(amenorrhea for 6-24 months and serum follicle-stimulating hormone level $>30 \mathrm{lU} / \mathrm{ml}$ ). Participants received either estrogen supplementation (estradiol group, $n=32$ ) or no supplementation (control group, $n=33$ ), and underwent $\mu \mathrm{MRI}$ of the distal radius and tibia, dual X-ray absorptiometry of the spine and hip, and peripheral quantitative CT at baseline, 12 months and 24 months.

Significant topological changes in trabecular architecture were observed at the distal tibia in control individuals after 12 months, in addition to significantly decreased BMD at the spine and hip. By contrast, no parameters changed significantly between baseline and 12 months in the estradiol group. Most of the parameters measured indicated significant deterioration in the control group and significant improvement in the estradiol group after 24 months.

The authors conclude that their findings provide direct evidence of trabecular bone changes in early menopause, and of the protective effect of estrogen supplementation. Their noninvasive $\mu \mathrm{MRI}$ technique might be useful for monitoring the efficacy of other bone-related interventions.

Original article Wehrli FW et al. (2008) In vivo magnetic resonance detects rapid remodeling changes in the topology of the trabecular bone network after menopause and the protective effect of estradiol. J Bone Miner Res 23: 730-740

\section{An autosomal-dominant GPR54 mutation implicated in central precocious puberty}

The kisspeptin-GPR54 signaling complex seems to be crucial to the activation of pulsatile gonadotropin-releasing hormone secretion, a key event that triggers pubertal onset. Lossof-function GPR54 mutations are associated with hypogonadotropic hypogonadism, so Teles and colleagues hypothesized that gainof-function mutations in GPR54 might cause central precocious puberty-most cases of which occur in girls and are considered idiopathic.

These researchers identified an autosomaldominant GPR54 mutation (which caused an Arg386Pro substitution in the receptor's carboxy terminal) in an adopted girl who had slowly progressive thelarche from birth, accompanied by other pubertal features after age 7 years. A diagnosis of idiopathic central precocious puberty was confirmed by her satisfactory response to depot leuprolide $(3.75 \mathrm{mg}$ per month). Analysis of DNA from this girl, 53 unrelated children with idiopathic central precocious puberty, and 150 ethnically matched healthy adult volunteers revealed no other individuals with this GPR54 mutation.

The Arg386Pro mutation did not alter GPR54 expression levels or its binding affinity for kisspeptin. However, kisspeptin stimulation of the mutant receptor caused markedly prolonged activation of downstream intracellular signaling pathways, indicative of nonconstitutive GPR54 activation. Mutant GPR54 also remained in cell membranes for longer after kisspeptin stimulation than wild-type receptor did, which indicated reduced receptor desensitization.

Teles and colleagues hypothesized that the increased response of Arg386Pro GPR54 to kisspeptin would cause release of increasedamplitude pulses of gonadotropin-releasing hormone, which might accelerate maturation of the reproductive axis and so cause central precocious puberty.

Original article Teles MG et al. (2008) A GPR54-activating mutation in a patient with central precocious puberty. $N$ Engl JMed 358: 709-715

\section{Increased glucose levels and blood pressure in IVF-conceived children}

Over the past years reports have suggested that adverse conditions during early prenatal life are associated with postnatal cardiometabolic dysfunction. Ceelen et al. retrospectively investigated whether children conceived following in vitro fertilization (IVF) were at increased risk of developing cardiometabolic problems.

The researchers compared 225 children born after IVF with a control group of 225 spontaneously conceived children matched for age and sex. All children (mean age at the time of followup 12.3 years, range 8-18 years) were born to parents with suboptimal fertility. All participants' blood pressure, skinfold thickness and body weight and height were recorded. A subset of 131 pubertal children from each group provided blood samples for measurement of indicators of insulin resistance.

Systolic and diastolic blood pressures, the sum of skin-fold thicknesses and fasting glucose levels were all significantly higher in children conceived following IVF than in controls. No significant differences were found between 